\title{
The coordinated effects of Apatinib and Tripterine on the proliferation, invasiveness and apoptosis of human hepatoma Hep3B cells
}

\author{
HUIHUI LI, YICHANG FAN, FAN YANG, LEI ZHAO and BANGWEI CAO \\ Department of Cancer Center, Beijing Friendship Hospital, Capital Medical University, Beijing 100050, P.R. China
}

Received October 11, 2017; Accepted March 22, 2018

DOI: $10.3892 / \mathrm{ol} .2018 .8656$

\begin{abstract}
As a novel vascular endothelial growth factor receptor-2 (VEGFR-2) tyrosine kinase inhibitor, Apatinib has exhibited antitumor effects in a variety of solid tumors. Extracts of Chinese herbal medicines have emerged as a promising alternative option to increase the sensitivity of patients to chemotherapeutics while alleviating side effects. The present study aimed to investigate the effects of Apatinib and the traditional Chinese herb Tripterine on the proliferation, invasion and apoptosis of human hepatoma Hep3B cells. The expression of VEGFR-2 in Hep3B cells was detected by western blotting and immunofluorescence assays. Hep3B cells were then divided into four different groups: Control group, Apatinib group, Tripterine group and Apatinib plus Tripterine group. The proliferation, invasion and apoptosis of these four groups of Hep3B cells were assessed by MTS, wound healing and Transwell assays, and flow cytometry, respectively. Finally, the levels of the proliferation-associated proteins phosphorylated protein kinase $\mathrm{B}(\mathrm{p}-\mathrm{Akt})$ and phosphorylated extracellular signal-regulated kinase ( $p-E R K)$ and the apoptosis-associated proteins cleaved Caspase-3 and B-cell lymphoma-associated X protein (Bax) were detected by western blotting. The proliferation, migration and invasion of Hep3B cells were significantly inhibited by Apatinib and Tripterine, compared with the control group $(\mathrm{P}<0.01)$. The inhibitory effect of the combination group was markedly stronger than that of the Apatinib and Tripterine groups. The downregulation of $\mathrm{p}$-Akt and p-ERK induced by Apatinib
\end{abstract}

Correspondence to: Professor Bangwei Cao, Department of Cancer Center, Beijing Friendship Hospital, Capital Medical University, 95 Yong An Road, Xicheng, Beijing 100050, P.R. China E-mail: oncology@ccmu.edu.cn

Abbreviations: Akt, protein kinase B; ERK, extracellular signal-regulated kinase; p-Akt, phosphorylated protein kinase B; p-ERK, phosphorylated extracellular signal-regulated kinase; VEGFR, vascular endothelial growth factor receptor

Key words: Apatinib, Tripterine, proliferation, invasiveness, apoptosis, Hep3B cells and Tripterine was further inhibited in the combination group $(\mathrm{P}<0.05)$, and the expression levels of Caspase-3 and Bax were also significantly increased in the combination group $(\mathrm{P}<0.05)$. The combination of Apatinib and Tripterine significantly inhibited the proliferation, migration and invasion ability and promoted the apoptosis of Hep3B cells by downregulating the expression of $\mathrm{p}$-Akt and $\mathrm{p}-\mathrm{ERK}$, and upregulating the expression of Caspase-3 and Bax.

\section{Introduction}

Liver cancer is now considered a common malignancy and the second leading cause of cancer-associated mortality worldwide among males (1). Hepatocellular carcinoma (HCC) is the most common type of liver cancer, accounting for $90 \%$ of all cases (2). Of all the available treatment methods, hepatectomy is the most effective treatment for $\mathrm{HCC}$; however, only $20 \%$ of patients are eligible for surgery (3). For the remaining $80 \%$ of patients with $\mathrm{HCC}$ who are at an advanced stage of disease, surgical resection is not a viable option, and systemic chemotherapy is the principal treatment option (4). However, due to multidrug resistance and adverse reactions from traditional chemotherapy agents, the effective rate of systemic chemotherapy is only $10-15 \%$ (5). With the development of basic biomedical research for HCC, molecular targeted therapy has become a novel regimen for the treatment of HCC (6). Sorafenib, at present, is the only FDA approved drug with the same first-line treatment position to chemotherapy for the systemic therapy of HCC, and is a multi-target inhibitor, which may provide more survival benefits for patients with non-resectable HCC (7). Although patients with HCC exhibit a positive response to sorafenib, resistance to sorafenib usually appears in 3.5 months and the median progression-free survival (mPFS) has become a major obstacle for the extension of the median overall survival (mOS) (8). Consequently, the discovery of novel therapeutic drugs for the treatment of HCC has become an urgent requirement. A novel antiangiogenic agent, Apatinib, is a highly selective inhibitor of vascular endothelial growth factor receptor-2 (VEGFR-2) tyrosine kinase and possesses 10 times more binding ability than sorafenib (9). It may block downstream signaling through highly selective competition with intracellular VEGFR-2 ATP binding sites and prevent VEGF-mediated endothelial cell migration and proliferation (10). In pre-clinical research, 
Apatinib was demonstrated to have an inhibitory effect on neovascularization and exhibited potential antitumor activity against a series of tumor cells (11). A phase III clinical trial demonstrated that Apatinib improved the mPFS and mOS of patients with advanced gastric cancer (12). Simultaneously, Phase II clinical trials confirmed the antitumor effect of Apatinib in numerous cancer types, including non-small cell lung cancer (NSCLC), breast cancer and HCC (13-15). In patients with HCC, a small sample clinical trial demonstrated that Apatinib improved OS (16). It appears to be a possible treatment strategy for patients with advanced HCC. However, the potential antitumor mechanism in HCC remains unclear. A number of studies (17-19) have demonstrated that monomer drugs, including Tripterine from the traditional Chinese medicine Tripterygium, exhibited strong antitumor activity against different types of tumors. Tripterine, also known as celastrol, is one of the main active components of Tripterygium and has been confirmed to have antitumor effects in vitro and in vivo $(20,21)$. However, whether the traditional Chinese medicine monomer drug Tripterine may improve the antitumor activities of Apatinib is unknown. Therefore, the present study was designed to elucidate the mechanism of the antitumor effect of Apatinib on HCC cells. In addition, the synergistic antitumor effect of Tripterine with Apatinib and the molecular mechanism will also be investigated.

\section{Materials and methods}

Cell lines and reagents. Human hepatoma Hep3B cells were obtained from the Liver Disease Experimental Center of Beijing Friendship Hospital affiliated, Capital Medical University (Beijing, China). HUVECs were purchased from ScienCell Research Laboratories, Inc. (San Diego, CA, USA). The cells were cultured in Dulbecco's modified Eagle's medium (DMEM; Corning Incorporated, Corning, NY, USA) supplemented with $10 \%$ fetal bovine serum (FBS; ExCell Biology, Shanghai, China) at $37^{\circ} \mathrm{C}$ in the presence of $5 \% \mathrm{CO}_{2}$. Apatinib was purchased from Jiangsu Hengrui Medicine Co., Ltd. (Lianyungang, China). Tripterine was purchased from Shanghai Aladdin Company Bio-Chem Technology Co., Ltd. (Shanghai, China). MTS was purchased from Promega Corporation (Madison, WI, USA). The Annexin V-FITC/PI Apoptosis Detection kit was purchased from Nanjing Kaiji Bio Tech Co., Ltd. (Nanjing, China). Antibodies against protein kinase B (Akt; A5523), phosphorylated Akt (p-Akt; AP0274), extracellular signal-regulated kinase (ERK; A11116) and phosphorylated ERK (p-ERK; AP0472) were purchased from ABclonal (ABclonal Biotech Co., Ltd., Woburn, MA, USA). Antibodies against Caspase-3 (9662S) and B-cell lymphoma 2-associated X protein (Bax; 5023S), Antibodies against VEGFR-2 (2479S) were purchased from Cell Signaling Technology, Inc. (Danvers, MA, USA). Antibodies against GADPH (41549) were purchased from Signalway Antibody LLC (College Park, MD, USA). The dilution factor of the antibodies was 1:1,000.

Human hepatoma Hep3B cells were assigned into four different groups: Control group, Apatinib group, Tripterine group and combination group. The treatments in the four groups were no drug for the control group, $30 \mu \mathrm{mol} / 1$ Apatinib for the Apatinib group, $2.5 \mu \mathrm{mol} / 1$ Tripterine for the Tripterine group, and $30 \mu \mathrm{mol} / 1$ Apatinib with $2.5 \mu \mathrm{mol} / 1$ Tripertine for the combination group.

Western blot analysis. Human hepatoma Hep3B cells were seeded onto 6-well plates. After $24 \mathrm{~h}$, the cells were harvested. Cells were lysed with radioimmunoprecipitation assay lysis buffer (Nanjing Kaiji Bio Tech Co., Ltd. Nanjing, China), premixed with phenylmethanesulfonyl fluoride (dilution, 1:100). The protein concentration of the cell extracts was quantified using a bicinchoninic acid assay. Equal amounts of protein (40-60 $\mu \mathrm{g})$ were separated by SDS-PAGE gels $(8 \%$, $10 \%$ or $12 \%$ ) and then electrotransferred onto polyvinylidene difluoride membranes, followed by blocking with bovine serum albumin (BSA, Beijing Solarbio Science \& Technology Co., Ltd., Beijing, China) at room temperature for $30 \mathrm{~min}$ and incubation with primary antibodies at $4{ }^{\circ} \mathrm{C}$ overnight. TBST $(0.025 \%)$ was used to wash the membrane three times. The membranes were incubated with the secondary antibody at room temperature for $1 \mathrm{~h}$. The bands were detected using an enhanced chemiluminescence kit (Thermo Fisher Scientific, Inc., Waltham, MA, USA), and signal intensities were analyzed with a Gel-pro 4.5 Analyzer (Media Cybernetics, Rockville, MD, USA).

Immunofluorescence staining. Hep3B cells were plated at a density of $3 \times 10^{4}$ cells per well in 12 -well plates with coverslips. The coverslips were washed three times in PBS and fixed with $4 \%$ formaldehyde solution at room temperature for $20 \mathrm{~min}$. Next, the coverslips were washed three times in PBS and incubated with a membrane-permeation solution (1 ml Triton X-100 in $100 \mathrm{ml} \mathrm{PBS}$ ) at room temperature for $10 \mathrm{~min}$. Following washing three times in PBS, the coverslips were placed into $2 \% \mathrm{BSA}$ at room temperature for $30 \mathrm{~min}$. The coverslips were then incubated with a primary VEGFR-2 antibody (dilution, 1:100) at $4^{\circ} \mathrm{C}$ overnight. The coverslips were washed three times with PBS and incubated at room temperature for $60 \mathrm{~min}$ with a horseradish peroxidase-conjugated goat anti-rabbit immunoglobulin G secondary antibody (L3012-1; 1:100; Signalway Antibody LLC, College Park, MD, USA). The coverslips were washed three times with PBS and the nuclei were stained with DAPI at room temperature for $5 \mathrm{~min}$. Finally, stained cells were visualized under a confocal laser-scanning microscope(magnification, $\mathrm{x} 40$; oil, $\mathrm{NA}=1.25$; zoom=3) TCS SP5 (Leica Microsystems GmbH, Wetzlar, Germany).

MTS assay. Hep3B cells in the logarithmic growth phase were collected and inoculated into 96-well plates. A total of $50 \mu \mathrm{l}$ DMEM was then added to $50 \mu \mathrm{l}$ cell suspension (containing $1 \times 10^{4}$ cells) per well to reach a final volume of $100 \mu \mathrm{l}$. After the cells were adhered to the plates, they were treated with different concentrations $(0,10,30,50$ and $100 \mu \mathrm{mol} / \mathrm{l})$ of Apatinib for $24 \mathrm{~h}$ (Fig. 1A). The final optimal concentration of Apatinib was $30 \mu \mathrm{mol} / 1$. Similarly, Hep3B cells were treated with different concentrations $(0,1,2.5,5$ and $10 \mu \mathrm{mol} / \mathrm{l})$ of Tripterine for $24 \mathrm{~h}$ (Fig. 1B). The final optimal concentration of tripterine was $2.5 \mu \mathrm{mol} / 1$. After determining the optimal concentration of the aforementioned two drugs, Hep3B cells were treated with Apatinib at a final concentration of $30 \mu \mathrm{mol} / 1$ and Tripterine at a concentration of $2.5 \mu \mathrm{mol} / 1$. Four duplicate wells were set for each sample. Dimethyl sulfoxide 
A

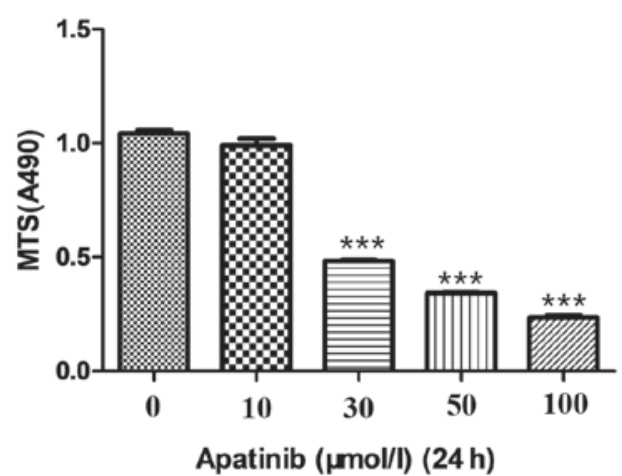

B

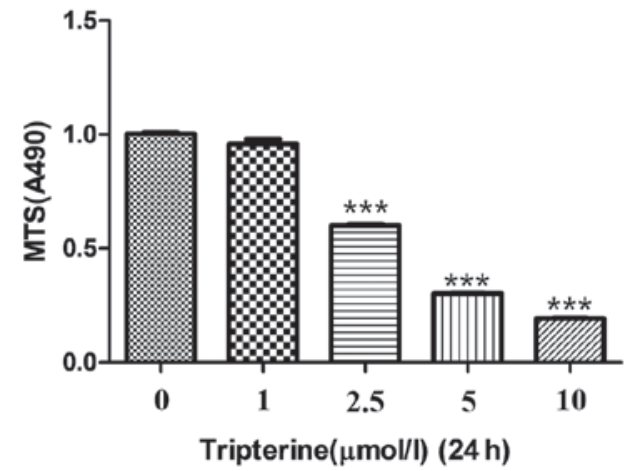

Figure 1. The inhibitory effects of Apatinib and Tripterine on the proliferation of Hep3B cells examined by MTS. (A) Hep3B cells were treated with with different concentrations $(0,10,30,50$ and $100 \mu \mathrm{mol} / 1)$ of Apatinib for $24 \mathrm{~h}$. (B) Hep3B cells were treated with different concentrations $(0,1,2.5,5$ and $10 \mu \mathrm{mol} / \mathrm{l})$ of Tripterine for $24 \mathrm{~h} .{ }^{* * *} \mathrm{P}<0.001 \mathrm{vs}$. control.

A

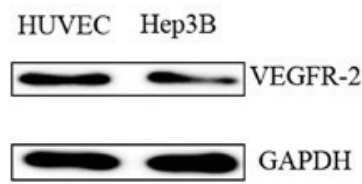

B

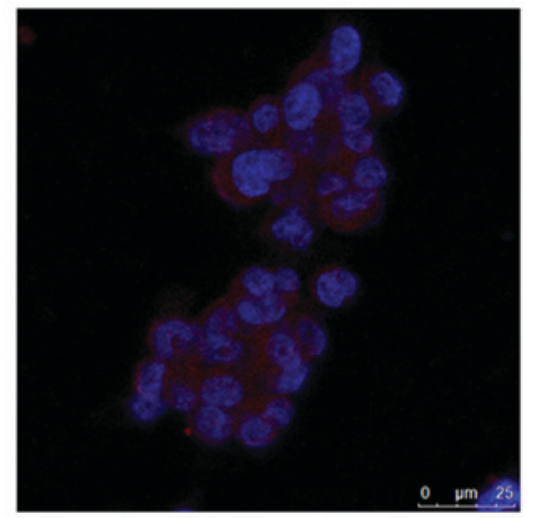

Figure 2. The expression of VEGFR-2 in Hep3B cells. (A) Representative western blot image of VEGFR-2 expression in Hep3B cells. (B) Representative immunofluorescence image of Hep3B cells to examine the localization of VEGFR-2 (Scale bar=25 $\mu \mathrm{m}$ ). VEGFR-2, vascular endothelial growth factor receptor-2. The blue fluoresence represents the nucleus and the red fluoresence represents the expression of VEGFR-2.

(50 $\mu \mathrm{l})$ was added to the blank control group. At the end of the experiment, $20 \mu \mathrm{l}$ MTS was added to each well and incubated at $37^{\circ} \mathrm{C}$ for $4 \mathrm{~h}$. Following aspiration of the supernatant, DMEM was added to the culture medium of each well. The OD value at a wavelength of $490 \mathrm{~nm}$ was measured using a microplate reader. The formula is cell proliferation rate (\%)=OD $490 \mathrm{~nm}_{\text {experimental group }} / \mathrm{OD} 490 \mathrm{~nm}_{\text {control group }} \mathrm{x} 100$. The inhibition rate $(\%)=\left(\right.$ average of OD $490 \mathrm{~nm}_{\text {control group }}$-average of OD $490 \mathrm{~nm}_{\text {experimental group }} /$ OD $490 \mathrm{~nm}_{\text {control group }}$ X100.

Wound healing assay. Hep3B cells were seeded at a density of $1 \times 10^{5}$ cells per well in 6 -well flat-bottomed microplates and then cultured to $90 \%$ confluency at $37^{\circ} \mathrm{C}$ in a humidified atmosphere with $5 \% \mathrm{CO}_{2}$. A thin scratch (wound) was made in the central area using pipette tips $(200 \mu \mathrm{l})$ and cells were carefully washed three times with DMEM. Different concentrations of Apatinib and Tripterine were then added to the culture medium of scratched Hep3B cells. Wound closure was monitored by light microscopy (magnification, x40). Images were acquired at 0,24 and $48 \mathrm{~h}$ post-scratching. The migration rate $(\%)=($ scratch distance-distance after growing $) /$ scratch distance.

Transwell assay. Matrigel diluted in cold serum-free DMEM was added to the bottom of the pre-cooled chamber prior to incubation at $37^{\circ} \mathrm{C}$. A total of $3 \times 10^{4} \mathrm{Hep} 3 \mathrm{~B}$ cells were added to the coated upper chamber containing DMEM. DMEM supplemented with $10 \%$ FBS was added to the lower chamber. After $24 \mathrm{~h}$ of incubation, the cells remaining on the upper chamber were wiped gently with cotton swab. Methanol (20\%) and $0.1 \%$ crystal violet at room temperature for $20 \mathrm{~min}$ were used to stain the cells that invaded through the membrane. Light microscopy was used to observe migrated cells in the lower chamber (magnification, x100).

Flow cytometry assay. Apoptotic cells were quantified by flow cytometry using an Annexin V-FITC/PI Apoptosis Detection kit (BD Biosciences, Franklin Lakes, NJ, USA), according to the manufacturer's protocol. Briefly, Hep3B cells were treated with $30 \mu \mathrm{mol} / 1$ Apatinib, $2.5 \mu \mathrm{mol} / 1$ Tripterine or a combination of $2.5 \mu \mathrm{mol} / 1$ Tripterine and $30 \mu \mathrm{mol} / 1$ Apatinib for 24 or $48 \mathrm{~h}$. Cells were then collected and washed twice with cold PBS followed by resuspension with $500 \mu$ l Annexin V binding buffer containing $5 \mu \mathrm{l}$ fluorescein isothiocyanate (FITC)-labeled Annexin V. The cell suspension was transferred into round-bottom tubes and incubated for $15 \mathrm{~min}$ in the dark at room temperature. Finally, $5 \mu \mathrm{l}$ propidium iodide (PI) was used and the percentage of apoptotic cells was measured using a flow cytometer and BD facs divaTM software (version 7.0; BD Biosciences). 

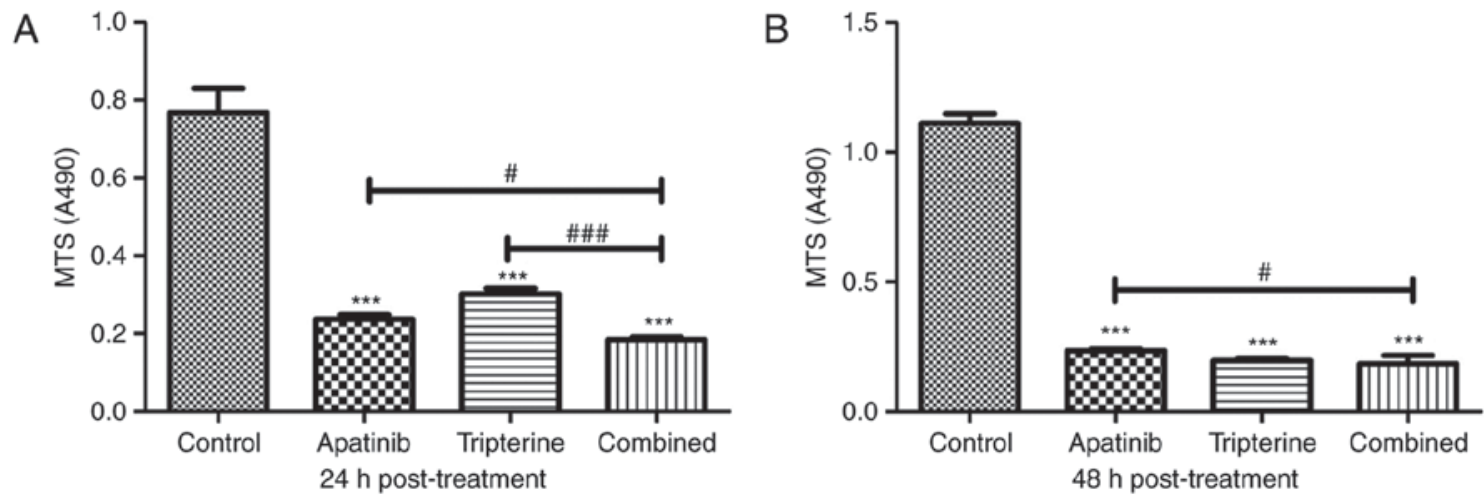

Figure 3. The inhibitory effects of Apatinib (30 $\mu \mathrm{mol} / \mathrm{l})$ and Tripterine $(2.5 \mu \mathrm{mol} / \mathrm{l})$ on the proliferation of Hep3B cells examined by MTS. (A) Hep3B cells were treated with various drugs for $24 \mathrm{~h}$. (B) Hep3B cells were treated with various drugs for $48 \mathrm{~h} .{ }^{* * * *} \mathrm{P}<0.001$ vs. control; ${ }^{\#} \mathrm{P}<0.05$ vs. combined treatment; ${ }^{\# \#} \mathrm{P} 0.001$ vs. combined treatment.

Statistical analysis. Data are presented as the mean \pm standard deviation. The differences between the control and experimental groups were analyzed by one-way analysis of variance, followed by the Student-Newman-Keul's (SNK) post hoc test. The statistical differences were evaluated by SPSS 19.0 software (IBM Corp., Armonk, NY, USA). P $<0.05$ was considered to indicate a statistically significant difference.

\section{Results}

The expression of VEGFR-2 in Hep3B cells. HUVECs were used as a positive control and VEGFR-2 was demonstrated to be expressed in Hep3B cells as detected by western blot analysis (Fig. 2A). An immunofluorescence assay also demonstrated that VEGFR-2 was located on the Hep3B cells (Fig. 2B). Therefore, VEGFR-2 was demonstrated to be expressed in Hep3B cells.

Tripterine enhances the inhibitory effect of Apatinib on Hep3B cell proliferation. An MTS assay was used to detect the effects of Apatinib, Tripterine and the combination of drugs on the proliferation of Hep3B cells. According to our pre-experiment, $30 \mu \mathrm{mol} / 1$ Apatinib combined with $2.5 \mu \mathrm{mol} / 1$ Tripterine may be the optimal therapeutic concentration for Hep3B cells (Fig. 1). At $24 \mathrm{~h}$ post-treatment, Apatinib, Tripterine and a combination of the two significantly inhibited the proliferation of Hep3B cells compared with the control group $(\mathrm{P}<0.001)$. The inhibitory effect of the combination group was more pronounced than that of the Apatinib and Tripterine groups (Fig. 3A). At $48 \mathrm{~h}$ post-treatment, the inhibitory effects were more evident in all three groups. However, the inhibitory capacity of the combination group was significantly stronger than that of the Apatinib group $(\mathrm{P}<0.05)$. However there was no significant difference in the combination group at $48 \mathrm{~h}$ post-treatment compared with the Tripterine group ( $\mathrm{P}>0.05$; Fig. 3B). The results demonstrated that the inhibition rates were 69.1 and $60.4 \%$ of Apatinib and Tripterine alone at $24 \mathrm{~h}$ post-treatment, respectively. However, following treatment with the combination of two drugs for $24 \mathrm{~h}$, the inhibition rate was as high as $75.8 \%$. At $48 \mathrm{~h}$ post-treatment, the inhibition rates of Apatinib and Tripterine were 78.8 and $82.2 \%$, respectively, while the inhibition rate of the combination of the two drugs was $83.5 \%$. Therefore, Tripterine enhanced the inhibitory effect of Apatinib on Hep3B cell proliferation.

The migration and invasion of Hep3B cells were inhibited by Apatinib and Tripterine. Identical drug concentrations were selected according to the MTS assay as previously described. Wound healing assays were performed to determine the migration effect of Hep3B cells under different drug treatments (Fig. 4A). A total of $24 \mathrm{~h}$ after scratching compared with the control group, Apatinib and Tripterine inhibited Hep3B cell migration; however, the difference was not statistically significant $(\mathrm{P}>0.05$; Fig. 4B). By contrast, the combination group significantly inhibited Hep3B cell migration $(\mathrm{P}<0.01)$. Compared with Apatinib or Tripterine alone, the combination group exhibited a significantly more evident inhibitory migration effect $(\mathrm{P}<0.01$ and $\mathrm{P}<0.05$, respectively; Fig. $4 \mathrm{~A}$ and $\mathrm{B})$. At $48 \mathrm{~h}$ post-treatment, the inhibitory effect of Hep3B cell migration was significantly enhanced in all three groups (all $\mathrm{P}<0.01$ ). Furthermore, the combination group and the Apatinib group exhibited a significantly stronger inhibitory migration effect $(\mathrm{P}<0.001$; Fig. 4A and $\mathrm{C}$ ). Transwell assays were performed to determine the invasive effect of Hep3B cells under different drug treatments. After $24 \mathrm{~h}$ of the Transwell assay, compared with the control group, all treatments significantly inhibited Hep3B cell invasion $(\mathrm{P}<0.001)$. The inhibitory effect was the strongest in the combination group and the difference was statistically significant compared with the Tripterine group $(\mathrm{P}<0.05$; Fig. 4D and E). Therefore Tripterine enhanced the inhibitory effect of Apatinib on Hep3B cell migration and invasion.

Tripterine combined with Apatinib promoted the apoptosis of Hep $3 B$ cells. The present study evaluated the effectiveness of the treatment in terms of apoptosis by performing Annexin V and PI staining on Hep3B cells from different treatment groups at 24 and $48 \mathrm{~h}$ after treatment. The present study identified a larger population of apoptotic cells in the Apatinib, Tripterine and combination groups at 24 and $48 \mathrm{~h}$ post-treatment $(\mathrm{P}<0.001$, respectively), while the pro-apoptotic effect of the combination treatment was evident compared with that of Apatinib or Tripterine treatment alone $(\mathrm{P}<0.05$, 

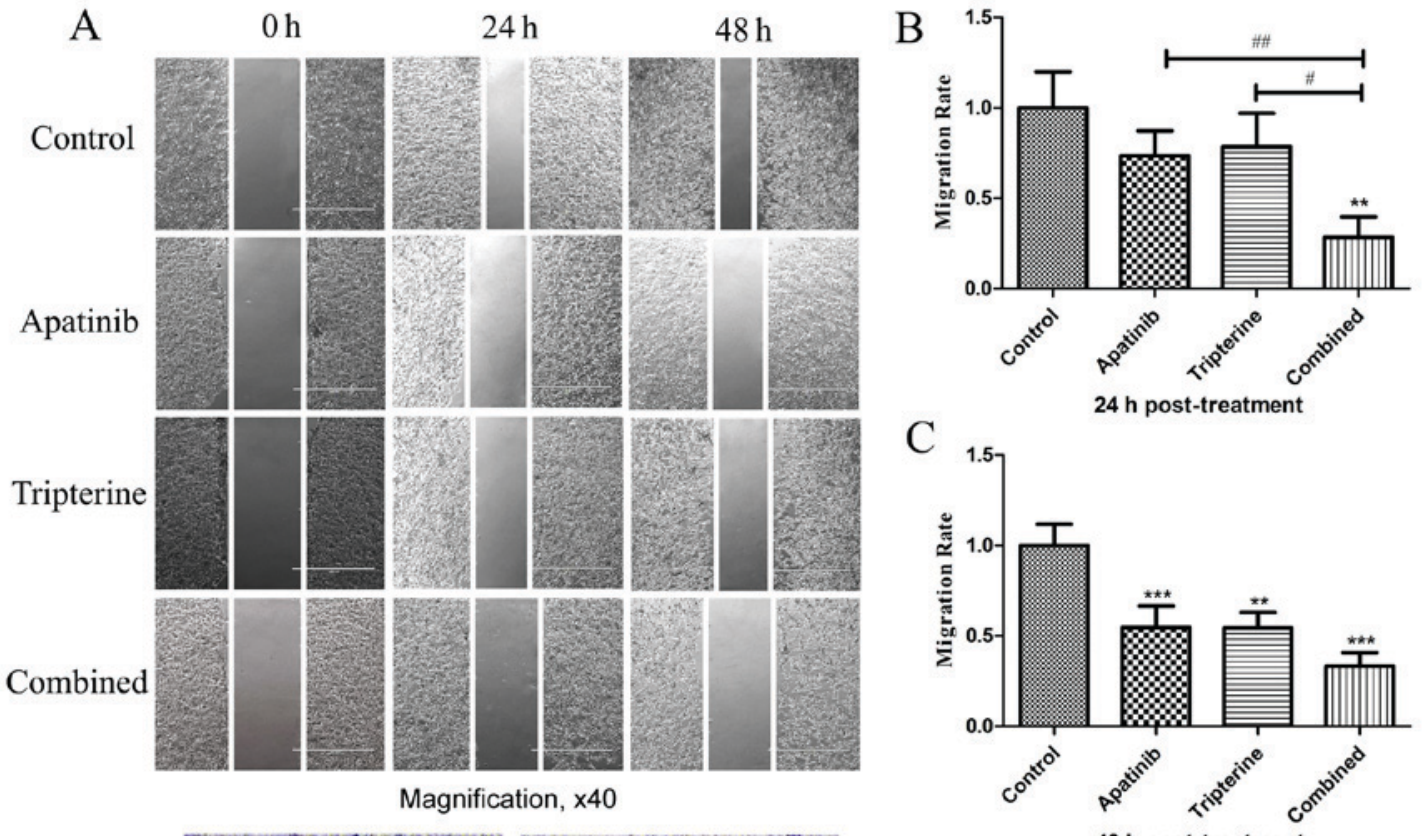

$\mathrm{D}$

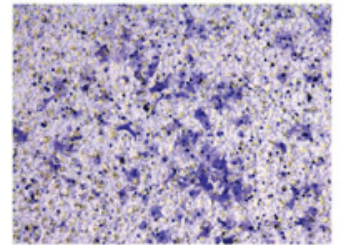

Control

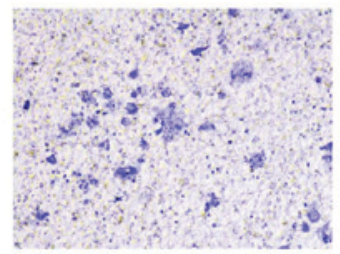

Tripterine

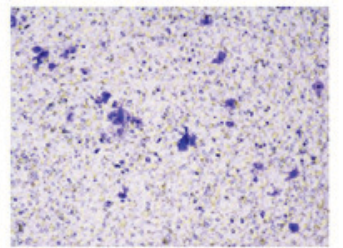

Apatinib

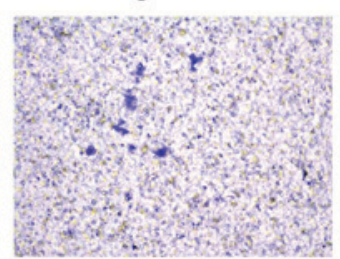

Combined

Magnification, $x 100$

Figure 4. The inhibitory effects of various drugs (Apatinib $30 \mu \mathrm{mol} / 1$ and Tripterine $2.5 \mu \mathrm{mol} / 1$ ) on the migration and invasion of Hep3B cells detected by wound healing and Transwell assays. (A) Wound healing assay (magnification, $\mathrm{x} 40$ ). Photomicrograph of wound width in Hep3B cells at 0,24 and $48 \mathrm{~h}$ after scratching. (B) Comparison of cell migration rates between the four groups at $24 \mathrm{~h}$ post scratching. (C) Comparison of cell migration rates between the four groups at $48 \mathrm{~h}$ post scratching. (D) Transwell assay. Cells were stained with crystal violet and subsequently captured by an inverted microscope (x100 magnification). (E) Quantification of cell migration rates between the four groups. ${ }^{* *} \mathrm{P}<0.01,{ }^{* * * *} \mathrm{P}<0.001,{ }^{\#} \mathrm{P}<0.05$ and ${ }^{\# \#} \mathrm{P}<0.01$.

respectively; Fig. 5A-D). Tripterine promoted the apoptosis of Hep3B cells via Apatinib.

Apatinib and Tripterine downregulated the expression of p-Akt and p-ERK and upregulated the expression of cleaved Caspase-3 and Bax. The present study demonstrated that the combination of Apatinib and Tripterine promoted the caspase-dependent apoptosis of Hep3B cells. As demonstrated in Fig. 6A and B, the expression of cleaved caspase- 3 and Bax was elevated in the Apatinib, Tripterine and combination groups, and the increases in cleaved caspase-3 and Bax were more evident in the combination group at 24 and $48 \mathrm{~h}$ post-treatment (Fig. 6C and D). ERK and Akt are downstream regulators of VEGFR-2 that serve a vital role in the process of apoptosis (22). Therefore, the expression of p-Akt and p-ERK were detected via western blotting. The results demonstrated that the expression of p-Akt and p-ERK were significantly decreased in the Apatinib, Tripterine and combination groups (Fig. 6A and C). The expression of p-Akt and p-ERK in the combination group was significantly lower than that in the Apatinib group or the Tripterine group (Fig. 6B and D). These findings suggested that the reduced phosphorylation of Akt and ERK may be the key to inducing caspase-dependent apoptosis in Hep3B cells. The combination of Apatinib and Tripterine downregulated the expression of p-Akt and p-ERK and upregulated the expression of cleaved Caspase-3 and Bax.

\section{Discussion}

A large volume of evidence has demonstrated that tumor angiogenesis is an essential event in the process of tumor growth and metastasis, which lead to the poor prognosis of 
A
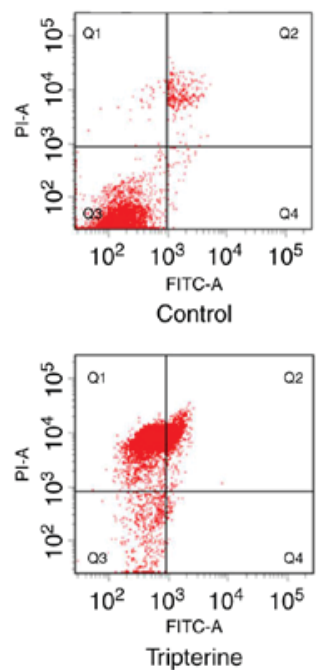

Tripterine
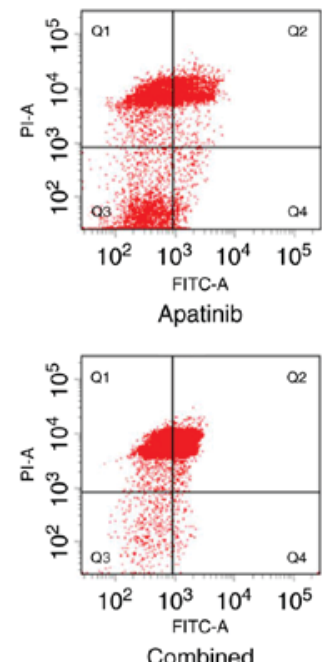

Combined

$24 \mathrm{~h}$ post-treatment

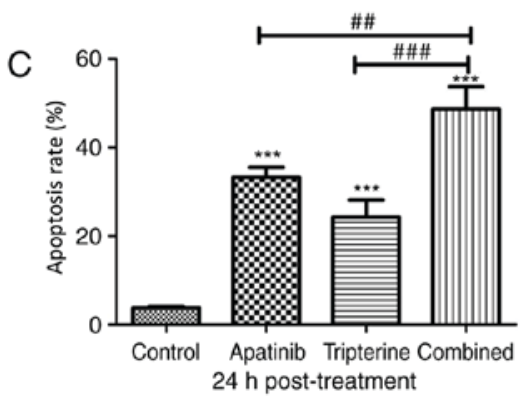

B
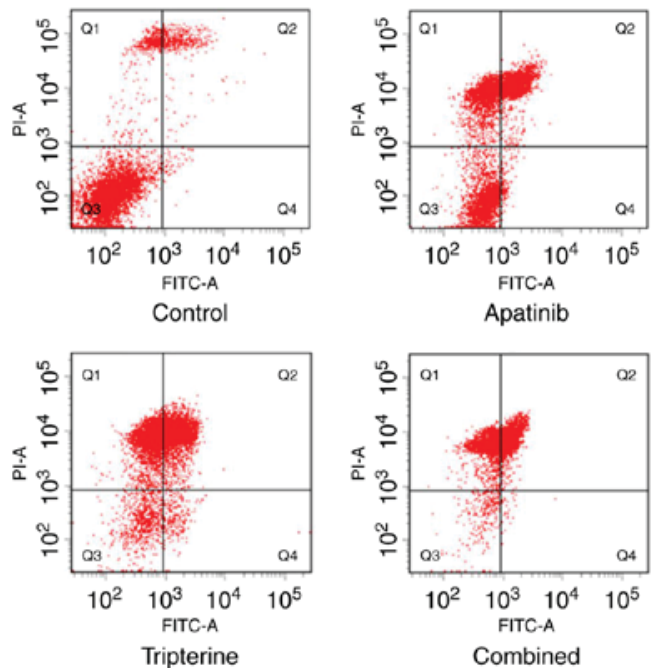

$48 \mathrm{~h}$ post-treatment

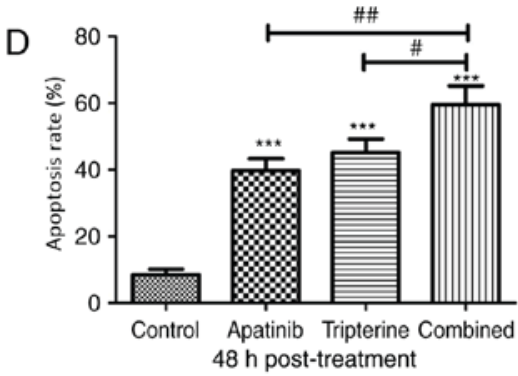

Figure 5. The pro-apoptotic effect of Apatinib (30 $\mu \mathrm{mol} / \mathrm{l})$ and Tripterine $(2.5 \mu \mathrm{mol} / \mathrm{l})$ on Hep3B cells was detected by flow cytometry. (A) Apoptosis rates of Hep3B cells after $24 \mathrm{~h}$ measured using flow cytometry with double staining by Annexin V and PI. (B) Apoptosis rates of Hep3B cells after 48 h measured using flow cytometry with double staining by Annexin V and PI. (C) The percentage of Q2\% plus Q4\% of different groups after 24 h. (D) The percentage of Q2\% plus Q4\% of different groups after 48 h. Q2, late-stage apoptosis rate; $\mathrm{Q} 4$, early-stage apoptosis rate. ${ }^{*} \mathrm{P}<0.05,{ }^{* *} \mathrm{P}<0.01,{ }^{* * * *} \mathrm{P}<0.001,{ }^{\#} \mathrm{P}<0.05,{ }^{\# \#} \mathrm{P}<0.01$ and

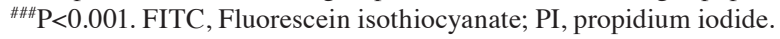

HCC (23-25). HCC becomes more aggressive and lethal once it obtains a sufficient blood supply (26). Therefore, it is of great importance to block angiogenesis in HCC. Among all angiogenic factors, VEGF is the key regulator that triggers a series of signaling pathways to promote endothelial cell proliferation, migration and survival from pre-existing vasculature (27). VEGF/VEGFR are highly expressed in the majority of tumor tissues. Therefore, the VEGF-VEGFR pathway has been a research hotspot in the field of anti-angiogenesis therapy $(28,29)$.

Apatinib is an orally administered small-molecule inhibitor that selectively targets VEGFR-2, inhibiting the activities of platelet-derived growth factor-b receptor, c-kit, and c-src, which subsequently suppress the formation of new blood vessels (30). Angiogenesis is an important mechanism of tumorigenesis (31). The study of Li et al (32) demonstrated that Apatinib significantly improved the survival outcomes of patients with gastric cancer who underwent second-line treatment failure in a phase II clinical trial, which was consistent with the research of Roviello et al (33). Therefore, the present study used Apatinib as a novel anti-tumorigenesis agent for the treatment of HCC.

Tripterine is a traditional medicine monomer extracted from the Chinese herb Tripterygium wilfordii HOOK $\mathrm{f}$. Previous studies have demonstrated that Tripterine has anti-inflammatory, anti-immune and antitumor effects (34-36).
Tripterine has been demonstrated to induce apoptosis in human triple-negative breast cancer cells by upregulating the expression of Bax and downregulating the activity of the phosphoinositide 3-kinase enzyme and the phosphorylation of Akt (17). Lee et al (18) also identified an increase in phosphorylated mitogen-activated protein kinase following a decrease in all phosphorylated forms of Akt, mechanistic target of rapamycin and S6K following treatment with Tripterine in gastric cancer. Therefore, the present study further investigated the synergistic antitumor effect of Apatinib and Tripterine. The proliferation, migration and invasion of tumor cells contribute toward the local infiltration and distant metastasis of malignancies, which subsequently lead to the lethality of cancer.

In the present study, the effects of Apatinib combined with Tripterine on the proliferation, migration and invasion of Hep3B cells were investigated. Due to the function of VEGFR-2 as a membrane receptor protein, western blot analysis and immunofluorescence assays were used to confirm the expression of VEGFR-2 in Hep3B cells. The results demonstrated that the proliferation, migration and invasion of HCC cells were significantly inhibited by Apatinib and Tripterine, and the inhibitory effect was more evident in the combination group, suggesting that Apatinib and Tripterine had a synergistic effect in anti-tumorigenesis. Furthermore, the underlying mechanism of this process was elucidated. As a key tumor suppression mechanism, apoptosis is a process of 

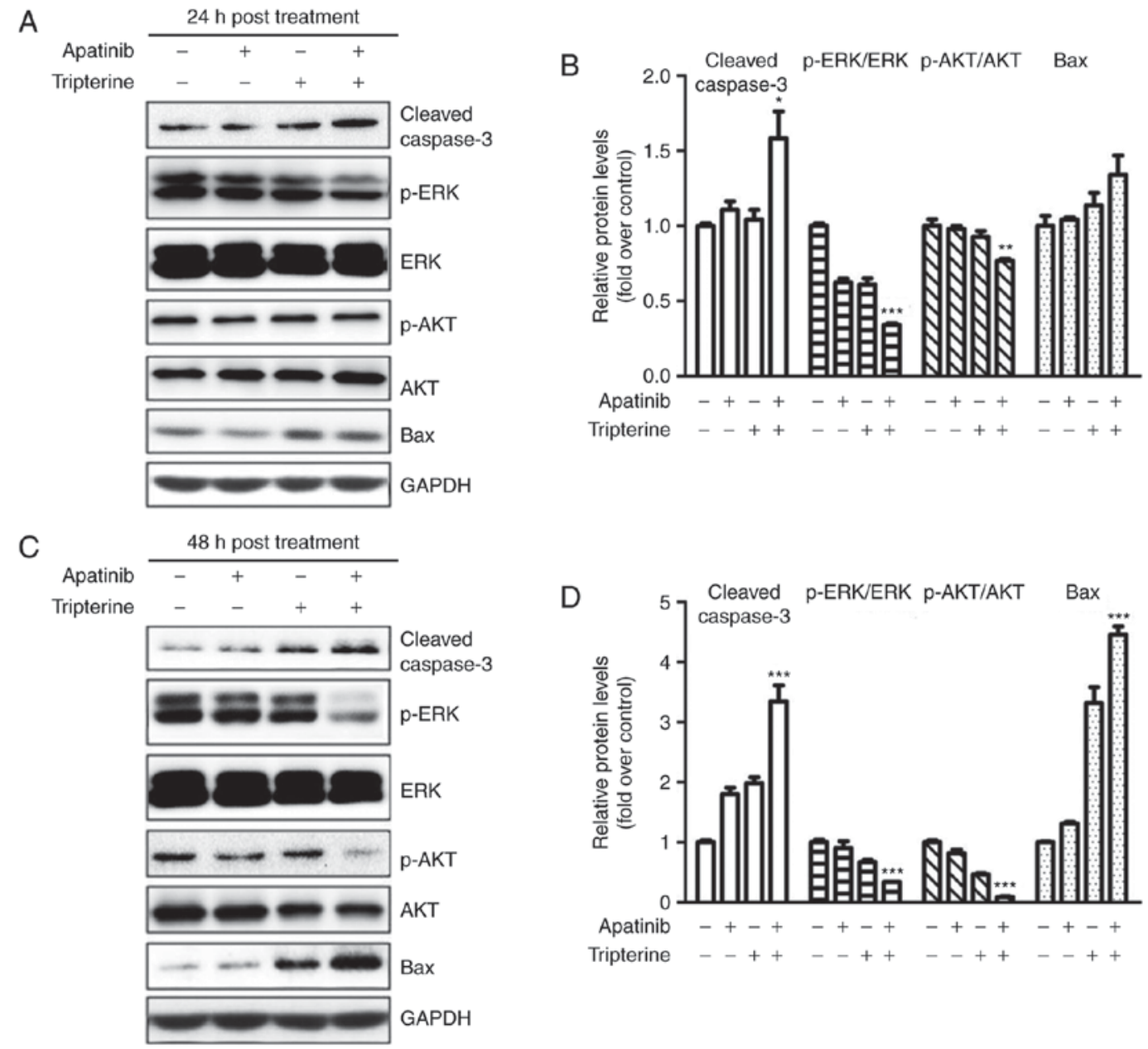

Figure 6. Protein expression in various treatment groups (Apatinib $30 \mu \mathrm{mol} / 1$ and Tripterine $2.5 \mu$ mol/1) detected by western blot analysis. (A) Expression of p-Akt, p-ERK, cleaved caspase-3 and Bax in four different groups at $24 \mathrm{~h}$ post-treatment. (B) Quantification of different protein expression levels between the four groups at $24 \mathrm{~h}$ post-treatment. (C) Expression of p-Akt, p-ERK, cleaved caspase-3 and Bax in four different groups at $48 \mathrm{~h}$ post-treatment. (D) Quantification of different protein expression levels between the four groups at $48 \mathrm{~h}$ post-treatment. ${ }^{*} \mathrm{P}<0.05$, ${ }^{* *} \mathrm{P}<0.01$ and ${ }^{* * *} \mathrm{P}<0.001$. Akt, protein kinase B; ERK, extracellular signal-regulated kinase; p-Akt, phosphorylated protein kinase B; p-ERK, phosphorylated extracellular signal-regulated kinase; Bax, B-cell lymphoma-associated X protein.

programmed cell death that can be initiated by the pro-apoptotic factor Bax. Bax is widely expressed in a variety of cells and is an important apoptotic protein $(37,38)$. Bax translocation from the cytosol to the outer mitochondrial membrane alters the permeability of the mitochondrial membrane and releases several pro-apoptotic factors, including cytochrome c (39). In addition to Bax, caspase-3 is a crucial mediator of apoptosis that catalyzes the specific cleavage of numerous key pro-apoptotic proteins that leads to DNA fragmentation and the formation of apoptotic bodies (40). The present study examined whether Apatinib and Tripterine could induce apoptosis of HCC and demonstrated that Apatinib and Tripterine could significantly increase apoptosis. Furthermore, apoptosis was more evident with a combination of the two drugs. According to the flow cytometry assay, the number of necrotic cells was large, culminating in the hypothesis that there were a number of other mechanisms for cell death in addition to apoptosis that require further research. The focus was on the effects of different drugs on the apoptosis of Hep3B cells. In addition, the expression of cleaved caspase- 3 and Bax was significantly increased in the Apatinib and Tripterine groups and further increased in the combination group.
The ERK signaling pathway serves a vital role in numerous cell functions that mediate different proliferation-related events, including apoptosis, autophagy and senescence (41). Akt is also a key apoptosis-associated protein that promotes cell survival via the inhibition of apoptosis $(42,43)$. The expression of two important proteins, Akt and ERK, which are downstream of VEGFR-2, was examined and it was revealed that the expression of p-Akt and p-ERK was decreased in the Apatinib and Tripterine groups and further decreased in the combination group at $48 \mathrm{~h}$ post-treatment.

A combination of Apatinib and Tripterine significantly inhibited the proliferation, invasion and migration of Hep3B cells while promoting caspase-dependent apoptosis. However, there are certain limitations to the present study. To begin with, the expression of Bax was examined during an apoptosis assay. As previously mentioned, Bax translocation from the cytosol to the outer mitochondrial membrane releases several pro-apoptotic factors (44); consequently, detecting cellular Bax localization will be more intuitive and representative and should be the focus of future study. The activation of Caspase- 3 (cleaved caspase-3) indicates the progression of apoptosis into an irreversible stage, which can irrefutably demonstrate 
that apoptosis occurs (45). In future experiments, a cleaved caspase-3 and caspase-3 precursor should be detected to further confirm the results. Furthermore, when the expression of VEGFR-2 in Hep3B cells was examined, the specific location of VEGFR-2 in Hep3B cells was not established. However, according to previous reports, VEGFR-2 should be located on the cell membrane (46). Future studies should use non-permealibilized cells as a control and a membrane marker to verify whether VEGFR-2 was expressed on the cellular membrane. In conclusion, the present study may represent a potential treatment strategy for patients in the advanced stages of liver cancer. However, more studies should be conducted in order to evaluate the safety and the appropriate dose in a clinical setting.

\section{Acknowledgements}

The authors would like to thank Dr Zhaoyu Zhong (Harbin Medical University, Harbin, China) for providing advice and technical assistance regarding the design of this study.

\section{Funding}

The present study was supported by grants from the Research Foundation of Beijing Friendship Hospital, Capital Medical University (grant no. yyqdkt 2015-11), the Beijing Natural Science Foundation (grant no. 7172081) and the Beijing Administration of Traditional Chinese Medicine (grant no. JJ2016-16).

\section{Availability of data and materials}

The datasets used and/or analyzed during the current study are available from the corresponding author on reasonable request.

\section{Authors' contributions}

$\mathrm{HL}$ and $\mathrm{BC}$ designed the study. The data were analyzed by HL, LZ, FY and YF. The figures were prepared by HL and LZ. HL wrote the paper. All authors reviewed the manuscript.

\section{Ethics approval and consent to participate}

Not applicable.

\section{Consent for publication}

Not applicable.

\section{Competing interests}

The authors declare that they have no competing interests.

\section{References}

1. Torre LA, Sauer AM, Chen MS Jr, Kagawa-Singer M, Jemal A and Siegel RL: Cancer statistics for Asian Americans, native Hawaiians, and Pacific Islanders, 2016: Convergence of incidence between males and females. CA Cancer J Clin 66: 182-202, 2016.

2. Molina-Sánchez P and Lujambio A: Strategies for HCC target discovery. Aging (Albany NY) 9: 1088-1089, 2017.
3. Azumi M, Suda T, Terai S, Terai K and Akazawa K: Prognostic impact of indocyanine green plasma disappearance rate in hepatocellular carcinoma patients after radiofrequency ablation: A prognostic nomogram study. Intern Med 56: 1001-1007, 2017.

4. Yu SJ: A concise review of updated guidelines regarding the management of hepatocellular carcinoma around the world: 2010-2016. Clin Mol Hepatol 22: 7-17, 2016.

5. Lin J, Shen A, Chen H, Liao J, Xu T, Liu L, Lin J and Peng J: Nitidine chloride inhibits hepatic cancer growth via modulation of multiple signaling pathways. BMC Cancer 14: 729, 2017.

6. Liu Z, Wang T, Zhang Z, Tang S, Feng S, Yue M, Hu M, Xuan L and Chen Y: Survivin downregulation using siRNA nanoliposomes inhibits cell proliferation and promotes the apoptosis of MHCC-97H hepatic cancer cells: An in vitro and in vivo study. Oncol Lett 13: 2723-2730, 2017.

7. Kelley RK, Verslype C, Cohn AL, Yang TS, Su WC, Burris H, Braiteh F, Vogelzang N, Spira A, Foster P, et al: Cabozantinib in hepatocellular carcinoma: Results of a phase 2 placebo-controlled randomized discontinuation study. Ann Oncol 28: 528-534, 2017.

8. Al-Rajabi R, Patel S, Ketchum NS, Jaime NA, Lu TW, Pollock BH and Mahalingam D: Comparative dosing and efficacy of sorafenib in hepatocellular cancer patients with varying liver dysfunction. J Gastrointest Oncol 6: 259-267, 2017.

9. Peng S, Zhang Y, Peng H, Ke Z, Xu L, Su T, Tsung A, Tohme S, Huang $\mathrm{H}$, Zhang $\mathrm{Q}$, et al: Intracellular autocrine VEGF signaling promotes EBDC cell proliferation, which can be inhibited by Apatinib. Cancer Lett 373: 193-202, 2016.

10. Roviello G, Ravelli A, Polom K, Petrioli R, Marano L, Marrelli D, Roviello F and Generali D: Apatinib: A novel receptor tyrosine kinase inhibitor for the treatment of gastric cancer. Cancer Lett 372: 187-191, 2016.

11. Li F, Liao Z, Zhao J, Zhao G, Li X, Du X, Yang Y and Yang J: Efficacy and safety of Apatinib in stage IV sarcomas: Experience of a major sarcoma center in China. Oncotarget 8: 64471-64480, 2017.

12. Li K and Li J: Current molecular targeted therapy in advanced gastric cancer: A comprehensive review of therapeutic mechanism, clinical trials, and practical application. Gastroenterol Res Pract 2016: 4105615, 2016.

13. Zhang H: Apatinib for molecular targeted therapy in tumor. Drug Des Devel Ther 9: 6075-6081, 2015.

14. Hu X, Cao J, Hu W, Wu C, Pan Y, Cai L, Tong Z, Wang S, Li J, Wang Z, et al: Multicenter phase II study of Apatinib in non-triple-negative metastatic breast cancer. BMC Cancer 14: $820,2014$.

15. Langer CJ, Mok T and Postmus PE: Targeted agents in the third-/fourth-line treatment of patients with advanced (stage III/IV) non-small cell lung cancer (NSCLC). Cancer Treat Rev 39: 252-260, 2013.

16. Kou P, Zhang Y, Shao W, Zhu H, Zhang J, Wang H, Kong L and Yu J: Significant efficacy and well safety of apatinib in an advanced liver cancer patient: a case report and literature review. Oncotarget 8: 20510-20515, 2017.

17. Shrivastava S, Jeengar MK, Reddy VS, Reddy GB and Naidu VG: Anticancer effect of celastrol on human triple negative breast cancer: Possible involvement of oxidative stress, mitochondrial dysfunction, apoptosis and PI3K/Akt pathways. Exp Mol Pathol 98: 313-327, 2015.

18. Lee HW, Jang KS, Choi HJ, Jo A, Cheong JH and Chun KH: Celastrol inhibits gastric cancer growth by induction of apoptosis and autophagy. BMB Rep 47: 697-702, 2014.

19. Rajendran P, Li F, Shanmugam MK, Kannaiyan R, Goh JN, Wong KF, Wang W, Khin E, Tergaonkar V, Kumar AP, et al: Celastrol suppresses growth and Induces apoptosis of human hepatocellular carcinoma through the modulation of STAT3/JAK2 signaling cascade in vitro and in vivo. Cancer Prev Res 5: 631-643, 2012.

20. Li H, Li Y, Liu D, Sun H and Liu J: miR-224 is critical for celastrol-induced inhibition of migration and invasion of hepatocellular carcinoma cells. Cell Physiol Biochem 32: 448-458, 2013.

21. Chakravarthy R, Clemens MJ, Pirianov G, Perdios N, Mudan S, Cartwright JE and Elia A: Role of the eIF4E binding protein $4 \mathrm{E}-\mathrm{BP} 1$ in regulation of the sensitivity of human pancreatic cancer cells to TRAIL and celastrol-induced apoptosis. Biol Cell 105: 414-429, 2013.

22. Yang $\mathrm{S}$ and Liu G: Targeting the Ras/Raf/MEK/ERK pathway in hepatocellular carcinoma. Oncol Lett 13: 1041-1047, 2017.

23. Popper HH: Progression and metastasis of lung cancer. Cancer Metastasis Rev 35: 75-91, 2016. 
24. Arvelo F, Sojo F and Cotte C: Tumour progression and metastasis. Ecancermedicalscience 10: 617, 2016.

25. Vilanova G, Colominas I and Gomez H: A mathematical model of tumour angiogenesis: Growth, regression and regrowth. J R Soc Interface 14: 20160918, 2017.

26. Fontanella C, Ongaro E, Bolzonello S, Guardascione M, Fasola G and Aprile G: Clinical advances in the development of novel VEGFR2 inhibitors. Ann Transl Med 2: 123, 2014.

27. Bertino G, Demma S, Ardiri A, Proiti M, Gruttadauria S, Toro A Malaguarnera G, Bertino N, Malaguarnera M, Malaguarnera M and Di Carlo I: Hepatocellular carcinoma: Novel molecular targets in carcinogenesis for future therapies. Biomed Res Int 2014: 203693, 2014.

28. Zhao Y and Adjei AA: Targeting angiogenesis in cancer therapy: Moving beyond vascular endothelial growth factor Oncologist 20: 660-673, 2015.

29. Al-Abd AM, Alamoudi AJ, Abdel-Naim AB, Neamatallah TA and Ashour OM: Anti-angiogenic agents for the treatment of solid tumors: Potential pathways, therapy and current strategies-A review. J Adv Res 8: 591-605, 2017.

30. Ding J, Chen X, Gao Z, Dai X, Li L, Xie C, Jiang H, Zhang L and Zhong D: Metabolism and pharmacokinetics of novel selective vascular endothelial growth factor receptor-2 inhibitor apatinib in humans. Drug Metab Dispos 41: 1195-1210, 2013.

31. Schulte N, Ebert MP and Härtel N: Gastric cancer: New drugs-New strategies. Gastrointest Tumors 1: 180-194, 2014.

32. Li J, Qin S, Xu J, Guo W, Xiong J, Bai Y, Sun G, Yang Y, Wang L, Xu N, et al: Apatinib for chemotherapy-refractory advanced metastatic gastric cancer: Results from a randomized, placebo-controlled, parallel-arm, phase II trial. J Clin Oncol 31: 3219-3225, 2013.

33. Roviello G, Ravelli A, Fiaschi AI, Cappelletti MR, Gobbi A Senti C, Zanotti L, Polom K, Reynolds AR, Fox SB and Generali D: Apatinib for the treatment of gastric cancer. Expert Rev Gastroenterol Hepatol 10: 887-892, 2016.

34. Venkatesha SH and Moudgil KD: Celastrol and its role in controlling chronic diseases. Adv Exp Med Biol 928: 267-289, 2016.

35. Wang Z, Zhai Z and Du X: Celastrol inhibits migration and invasion through blocking the NF- $\mathrm{B}$ pathway in ovarian cancer cells. Exp Ther Med 14: 819-824, 2017.

36. Figueiredo SAC, Salvador JAR, Cortés R and Cascante M: Novel celastrol derivatives with improved selectivity and enhanced antitumour activity: Design, synthesis and biological evaluation. Eur J Med Chem 138: 422-437, 2017.
37. Brahmbhatt H, Uehling D, Al-Awar R, Leber B and Andrews D: Small molecules reveal an alternative mechanism of Bax activation. Boichem J 473: 1073-1083, 2016.

38. Luna-Vargas MPA and Chipuk JE: Physiological and pharmacological control of BAK, BAX, and beyond.trends. Cell Biol 26: 906-917, 2016.

39. Todt F, Cakir Z, Reichenbach F, Emschermann F, Lauterwasser J, Kaiser A, Ichim G, Tait SW, Frank S, Langer HF and Edlich F: Differential retrotranslocation of mitochondrial Bax and Bak. EMBO J 34: 67-80, 2015.

40. Burgess JT, Bolderson E, Adams MN, Baird AM, Zhang SD, Gately KA, Umezawa K, O'Byrne KJ and Richard DJ: Activation and cleavage of SASH1 by caspase-3 mediates an apoptotic response. Cell Death Dis 7: e2469, 2016.

41. Kochetkova EY, Blinova GI, Bystrova OA, Martynova MG, Pospelov VA and Pospelova TV: Targeted elimination of senescent Ras-transformed cells by suppression of MEK/ERK pathway. Aging (Albany NY) 9: 2352-2375, 2017.

42. Adlung L, Kar S, Wagner MC, She B, Chakraborty S, Bao J, Lattermann S, Boerries M, Busch H, Wuchter P, et al: Protein abundance of AKT and ERK pathway components governs cell type-specific regulation of proliferation. Mol Syst Biol 13: 904 , 2017.

43. Ewald F, Nörz D, Grottke A, Bach J, Herzberger C, Hofmann BT, Nashan B and Jücker M: Vertical targeting of AKT and mTOR as well as Dual targeting of AKT and MEK signaling is synergistic in hepatocellular carcinoma. J Cancer 6: 1195-1205, 2015.

44. Renault TT and Chipuk JE: Death upon a kiss: Mitochondrial outer membrane composition and organelle communication govern sensitivity to BAK/BAX-dependent apoptosis. Chem Biol 21: 114-123, 2014.

45. McIlwain DR, Berger T and Mak TW: Caspase functions in cell death and disease. Cold Spring Harb Perspect Biol 7: a008656, 2013.

46. Wu W, Zhang D,Pan D,Zuo G, Ren X and Chen S: Downregulation of vascular endothelial growth factor receptor-2 under oxidative stress conditions is mediated by $\beta$-transduction repeat-containing protein via glycogen synthase kinase-3 $\beta$ signaling. Int J Mol Med 37: 911-920, 2016.

This work is licensed under a Creative Commons Attribution-NonCommercial-NoDerivatives 4.0 International (CC BY-NC-ND 4.0) License. 